\title{
Fundamental Capacity Analysis for Identically Independently Distributed Nakagami-q Fading Wireless Communication
}

\author{
Siam Bin Shawkat ${ }^{1}$, Md. Mazid-Ul-Haque ${ }^{2}$, Md. Sohidul Islam ${ }^{3}$, Borshan Sarker Sonok ${ }^{4}$ \\ Department of Computer Science \\ American International University-Bangladesh
}

\begin{abstract}
With the advancement in technology, decent transfer rate of data for fast communication is an exigency. Different distributions on different wireless communication channels have been used previously to model them and to do performance analysis on the systems. In this work, capacity analysis of identically independently distributed Nakagami-q fading single-input multiple-output (SIMO) wireless communication is presented. The derivation of channel capacity with the analytical solution have been conducted using small limit argument approximation. Where the small limit argument approximation corresponds to the low signal-to-noise ratio (SNR) regime. SIMO channel capacity behavior with respect to number of receiver antennas and with respect to SNR have been explored in depth. The improvement of capacity is depicted rigorously. It has been found that using Nakagami-q distribution, capacity of the system increases as number of receiver antenna increases. It is also found that the capacity of this SIMO wireless system can be further improved through changing of certain parameters.
\end{abstract}

Keywords-Wireless communication; SIMO channel capacity; Nakagami-q fading; Hoyt distribution; low SNR regime

\section{INTRODUCTION}

Wireless technologies have become an indivisible part of the daily life of a person in this digitized era. Wireless network with high capacity and high data rates is always wanted by everyone. A very important consideration is how fast data can be sent over a channel. Previously the main focus was to overcome the distortion of the received wireless signal. As technologies are increasing day by day, flawless high data transaction rate is now a big concern. Good channel capacity is one of the challenging issues in wireless communication. As a major need, channel capacity has to be improved as much as possible.

Shanon [1] developed the theorem which tells the maximum rate at which information can be transmitted over a communications channel of a specified bandwidth in the presence of noise. This theorem also specifies many important factors of communication channels. Since then many researchers have focused on analyzing and improving channel capacity on various distributions. These researches also involved systems like single-input single-output (SISO), single-input multiple-output (SIMO), multiple-input single-output (MISO), multiple-input multiple-output (MIMO). Authors in [2], discussed and showed that using multiple antennas at transmitter and receiver sides, higher capacity with efficiency can be attained. They showed that capacity increase has linear relation with the increase of antennas. In [3], authors presented comparisons between SISO and MIMO system channels and also analyzed capacities in various distributions which involved Uniform distribution, Chisquare distribution, Gaussian distribution in the SISO system. Authors worked in Rayleigh fading single-input multipleoutput (SIMO) channel system considering the presence of eavesdropper and found optimal power allocation at the side of transmitters in [4]. They have also described their proposed mathematical expression of secure outage probability. In [5], authors have examined both Broadcast channel and SISO channel in light communication system and used entropy power inequity and Lagrangian function to develop closed form lower and upper bound. They have also investigated the beamforming design problem of $\mathrm{BC}$ and VLC based on the utilization of obtained closed-form expression [5]. In [6], authors focused on increasing the data rate capacity of multiple-input multipleoutput (MIMO) system channels through comparison of SISO, SIMO and MISO systems. Experimental simulated results of SISO and MIMO are analyzed, discussed and compared [6]. Based on given error probability and block length, authors in [7], investigated maximal achievable data rate over quasi static SIMO channels. They have also verified the fast convergence to outage capacity through zero dispersion when there is an increase in the block length. Authors in [8], analyzed discrete time Rayleigh fading capacity in SISO \& MIMO systems, presented and discussed their results obtained from their findings. In [9], authors studied SISO and SIMO channels and measured frequency response of line-of-sight (LOS) and non-line-of-sight (NLOS) channels. Their measurement result indicates that high data rates can be supported by channel capacity and it depends on the distance of the transmitter and the receiver. They also found in their measurement in [9], that the relationship between SIMO system channel capacity and number of receiving antennas is not linear. Considering the presence of an external eavesdropper, authors in [10], studied confidential communication in gaussian MIMO channel with a number of receivers. They also focused on proving the secrecy capacity of MIMO channels which are degraded based on some important factors. Researchers focus on various distribution models for analyzing and developing SISO, SIMO, MISO, MIMO channels. Gaussian or normal distribution,

Rician, Nakagami-m, Nakagami-n, Rayleigh are very popular distribution models. Beside these models, Nakagami-q is one of them and has a wide area of research interest. A fading distribution, Nakagami-q or also known as Hoyt distribution, serves as a suitable decent model under incontrovertible conditions. Using this distribution model, authors in [11], 
presented a simulation program where envelopes of received signals can be modelled. They found that this modeling can be done without presiding over NLOS [11]. In [12], authors showed that it is possible to construct Hoyt or Nakagami-q distribution from a conditional exponential distribution model. They proposed a method that is able to analyze performances of any wireless link under Nakagami-q fading in a very convenient approach. Based on the derivation of the squared Hoyt distribution formula, authors in [13], derived simple expressions of secondary link capacity on various scenarios of their research interests. For severe fading, they showed that the capacity of ST-SR link increases in the presence of severe fading. Authors used this Hoyt distribution to model and analyze limit of SISO wireless communication channel's data rate in [14].

In this work, capacity analysis of SIMO wireless system over the identically independently distributed Nakagami-q fading wireless channel is presented. This study follows the derivation of the channel capacity as described in [14] and modifies the system model and channel capacity equations to fit this SIMO wireless system of this study. First, the derivation of SIMO channel capacity equation is done for low SNR regime. Then the analysis of capacity is made with respect to the number of antennas at the receiver end while also depicting an improvement in the capacity. Capacity corresponding to the instantaneous SNR of this system and a comparison of the capacity of SIMO system and SISO system are also shown.

The rest of the paper is structured as follows. In Section 2, Nakagami-q/Hoyt distribution. In Section 3, SIMO system model for this research work. Calculation of capacity for low SNR regimes for this Nakagami-q fading channels in Section 4. Analysis and results is Section 5. At the end, Section 6 includes the conclusions and future work of this study.

\section{NAKAGAMI-Q/HOYT DistRIBUTION}

Throughout the long periods of remote correspondences, and relying upon framework working situations, an extraordinary number of channel models have been proposed to depict the measurements of the plentifulness and period of multipath signals. Nakagami-q is one of the popular proposed distribution models. This model was presented by Nakagami as an estimation for Nakagami-m distribution within the scope of fading that stretches out from one-sided Gaussian model to the Rayleigh model [15]. The distribution model is being utilized all the more habitually in execution investigation and different examinations identified with mobile radio interchanges. In [16], authors found that this model can be easily used in mobile communication channels. This fading is generally seen in satellite connection subject to amazing ionospheric flash and strongly shadowed conditions [16]. Nakagami-q or Hoyt model is normally used to delineate the transient sign subject to fading in variety of certain mobile communication channels [17]. It's probably distribution function (PDF) [18] is where, $\mathrm{q}$ is the fading parameter of Nakagami-q distribution with the value range from 0 to 1 . Instantaneous SNR is represented as $\gamma$ and average SNR is represented as $\bar{\gamma}$. $I_{0}($. is represented as the modified Bessel function of the first kind of zeroth-order [14]. When $q=0$, it represents the one sided Gaussian fading and Rayleigh fading is represented by $\mathrm{q}=1$.

\section{SIMO SYSTEM MODEL}

The single-input multiple-output (SIMO) frequently used to empower a collector framework that gets signals from various free sources to scrap the impacts of fading. It has been utilized for a long time with short wave listening/accepting stations to cope with the impacts of ionospheric impedance and fading. The single-input multiple-output (SIMO) is the model where the transmitter includes a single antenna and the receiver end has numerous receiving antennas.

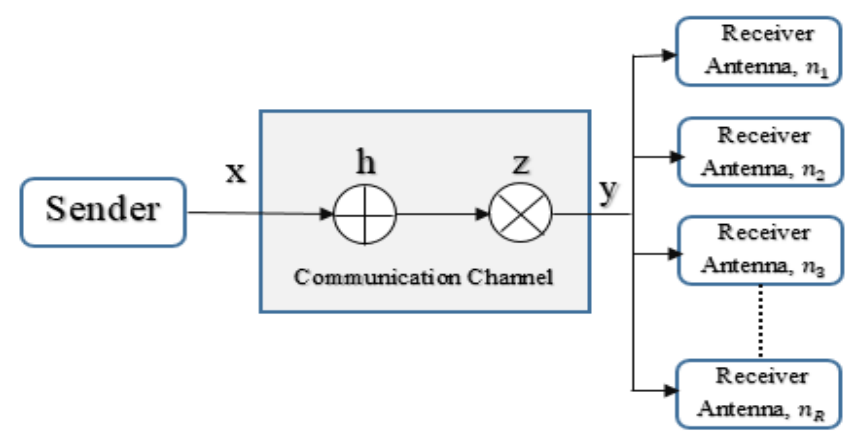

Fig. 1. Nakagami-q Fading SIMO Wireless Channel System Model.

Fig. 1 represents the SIMO system model for this research work considering the fact that the receiving power of each antennas are identical and the receiver antennas are mutually independent. Here, other considering factors include $P_{t}$ as transmitted signal power, signal getting corrupted by additive white gaussian noise (AWGN) at the receiving end and the transmission is continued with Nakagami-q distribution. Based on [19] received signal vector of this system is given by,

$$
r=h x+z
$$

In Eq. (2), channel gain from the transmitter to the receiver end is defined by $\mathrm{h}$ which is a vector, $\mathrm{x}$ as transmitted signal and complex additive white gaussian noise is represented by the vector z [19]. For SIMO systems, [20] states, Shannon channel limit is the most aloft responded data between the signal that is being sent and being received one. From [20], the equation which defines capacity is given as following,

$$
C=W \log _{2}(1+\rho)
$$

$$
p_{\gamma}(\gamma)=\frac{\left(1+q^{2}\right) \gamma}{2 q \bar{\gamma}} e^{\frac{-\left(1+q^{2}\right)^{2} \gamma}{4 q^{2} \bar{\gamma}}} I_{0}\left(\frac{\left(1-q^{4}\right) \gamma}{4 q^{2} \bar{\gamma}}\right), \quad \gamma \geq 0
$$

In Eq. (3), transmitted signal to noise ratio is represented as $\rho=P_{t} / \sigma^{2}$ and transmission bandwidth is represented as W. 
In [21] and [22] it has been said that, the channel is limited by power in the insight that, $P_{t}=E\left\{|x|^{2}\right\}$. Here, the E $\{$. denotes the expectation operator. This expectation operator can be assessed by the probability distribution function (PDF) of the above mentioned vector $h$.

Here, for low SNR regime, low limit argument approximation is considered as it is described by the author that, using low limit argument approximation, the zeroth-order modified Bessel function of the first kind can be estimated as $I_{0} \approx 1$ in [17].

So, using $I_{0} \approx 1$ in Eq. (1), it becomes,

$$
p_{\gamma}(\gamma)=\frac{\left(1+q^{2}\right) \gamma}{2 q \bar{\gamma}} e^{\frac{-\left(1+q^{2}\right)^{2} \gamma}{4 q^{2} \bar{\gamma}}}, \gamma \geq 0
$$

So, for low SNR regime, Nakagami-q distribution under the condition of low limit argument approximation is represented by Eq. (4). By changing fading parameter to q=1, Eq. (4) turns to the Rayleigh fading as described in the Nakagami-q/Hoyt Distribution section.

For SIMO channel, all the receiver antennas will be receiving fading signals, so the Nakagami-q fading parameter becomes, $q=n_{R} * q_{0}$, where number of antennas at the receiver end are represented as $n_{R}$ and when $n_{R}=1$ then the fading parameter, q becomes, $1 * q_{0}=q_{0}$, so the system becomes single-input single-output.

So, for SIMO channel Eq. (4) becomes,

$$
p_{\gamma}(\gamma)=\frac{\left(1+\left(n_{R} q_{0}\right)^{2}\right) \gamma}{2 n_{R} q_{0} \bar{\gamma}} e^{\frac{-\left(1+\left(n_{R} q_{0}\right)^{2}\right)^{2} \gamma}{4\left(n_{R} q_{0}\right)^{2} \bar{\gamma}}}, \gamma \geq 0
$$

For simplifying Eq. (5) let us consider

$$
x=\frac{1+\left(n_{R} q_{0}\right)^{2}}{2 n_{R} q_{0} \bar{\gamma}}, y=\frac{\left(1+\left(n_{R} q_{0}\right)^{2}\right)^{2}}{4\left(n_{R} q_{0}\right)^{2} \bar{\gamma}}
$$

Using the simplifications of Eq. (6), Eq. (5) becomes,

$$
p_{\gamma}(\gamma)=x \gamma e^{-y \gamma}, \quad \gamma \geq 0
$$

Here, Eq. (7) represents Nakagami-q distribution for for low SNR regime under low limit argument approximation [14].

\section{Calculation of Capacity}

The calculation of capacity in low SNR region for this SIMO wireless channel is done in this section. The capacity can be acquired a by using Eq. (3) [14],

$$
C=\int_{0}^{\infty} p_{\gamma}(\gamma) \log _{2}(1+\rho \gamma) d \gamma
$$

Performing substitution of Eq. (7) into the Eq. (8), the channel capacity of SIMO channel becomes,

$$
C=\int_{0}^{\infty} x \gamma e^{-y \gamma} * \log _{2}(1+\rho \gamma) d \gamma
$$

An advanced computing system, Mathematica [23] of Wolfarm Research is used here to elucidate and to validate Eq. (9) in order to analyze the capacity of SIMO channel.

The channel capacity, using Eq. (9) results in,

$$
C_{S I M O}=\frac{x}{\rho^{2} \log [2]}\left(G_{2,3}^{3,1}\left(\left.\frac{y}{\rho}\right|_{-2,-2,0} ^{-2,-1}\right)\right)
$$

if $\operatorname{Re}[\rho]>0$ and if $\operatorname{Re}[\mathrm{b}]>0$.

Here, $x=\frac{1+\left(n_{R} q_{0}\right)^{2}}{2 n_{R} q_{0} \bar{\gamma}}, y=\frac{\left(1+\left(n_{R} q_{0}\right)^{2}\right)^{2}}{4\left(n_{R} q_{0}\right)^{2} \bar{\gamma}}$ and $G_{p, q}^{m, n}\left(\left.x\right|_{b_{q}} ^{a_{p}}\right)$ is the Meijer Gamma function [14] and $C_{S I M O}$ represents the capacity of SIMO channel.

In the next section, Eq. (10) is used for the analysis of the SIMO channel capacity. The analyses are conducted with respect to the number of receiver antennas, average SNR, instantaneous SNR of the channel and the comparison of the capacity of SIMO and SISO systems are depicted also.

\section{ANALYSIS OF CAPACITY}

The analyses of SIMO channel capacity are presented here. The section is divided into four subsections. Capacity behaviour with respect to the number of receiver antennas, $n_{R}$ is presented in subsection one. For the improvement of the capacity of the system, capacity behaviour with respect to the number of receiver antennas, $n_{R}$ while varying the average SNR, $\bar{\gamma}$ of this system is presented in the next subsection. The following subsection includes capacity behaviour with respect to the instantaneous SNR of the system. And the last subsection contains a comparison between SIMO channel capacity of this research work and SISO channel capacity, which was depicted in [14].

For the analysis section the value for fading parameter $q_{0}$ is considered 0.17 because it has been found in this analysis that when $q_{0}>0.17$, the conditions of Eq. (10) become false.

\section{A. SIMO Channel Capacity with respect to the Number of Receiver Antennas}

This subsection describes the channel capacity of the Nakagami-q fading SIMO channel. The channel capacity depicted by Eq. (10) is plotted with respect to the number of antennas at the receiver end.For this, value of $n_{R}$ taken from 2 to 6 . And the value of the average SNR is taken $\bar{\gamma}=5$. The verification of the correctness of Eq. (10) is done also which has been procured from Eq. (9).

It is observed in Fig. 2 that the Eq. (10) is the correct solution of Eq. (9) as both equations have been plotted considering the same values and both the equations yields the exact same plot. So, the correctness of the SIMO channel capacity equation is justified.

From Fig. 2, it can be seen that the capacity is increasing with the increasing of the antennas at the receiver end. When there are 2 antennas at the receiver end the value of capacity of 


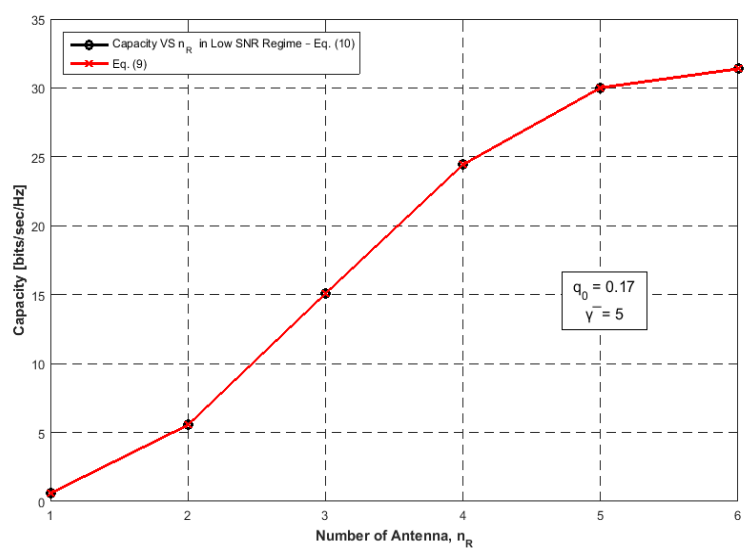

Fig. 2. SIMO Channel Capacity [bits/sec/Hz] with respect to the Number of Receiver Antennas, $n_{R}$.

is $5.54478 \mathrm{bits} / \mathrm{sec} / \mathrm{Hz}$, when $n_{R}=4$ the capacity is 24.4357 bits $/ \mathrm{sec} / \mathrm{Hz}$ and at last for $n_{R}=6$, the channel capacity is at it's highest which is $31.3864 \mathrm{bits} / \mathrm{sec} / \mathrm{Hz}$.

So, it is clear that with the increasing of the antennas at the receiver end the capacity of the this system is increasing.

\section{B. Improvement of SIMO Channel Capacity}

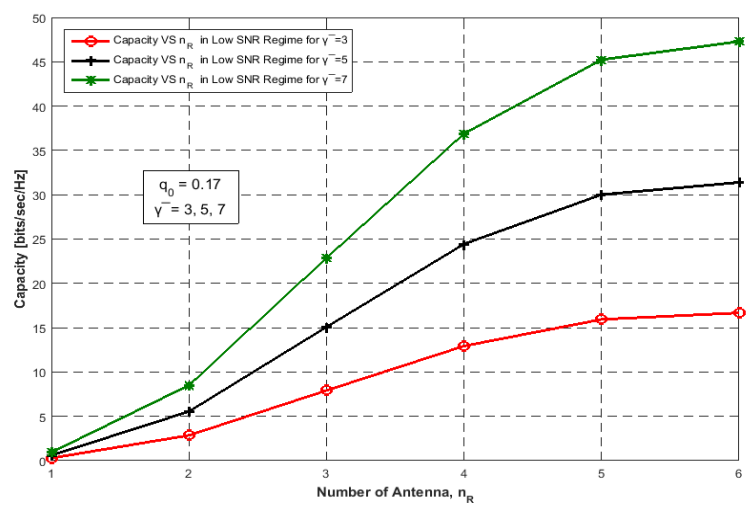

Fig. 3. Capacity [bits/sec/Hz] with respect to Number of Receiver Antennas, $n_{R}$ when $\bar{\gamma}$ varies

In this subsection again the capacity behaviour with respect to the number of receiver antennas are presented but varying the average SNR, $\bar{\gamma}$ of the system. As in the previous subsection here also the number of receiver antennas are from 2 to 6 . The value of average SNR of the system is taken, $\bar{\gamma}=3,5,7$ respectively for the analysis of this subsection.

It can be observed in Fig. 3 that for all the three cases the channel capacity is increasing when the number of receiver antenna increases. But, it can also be observed that the capacity increase is even higher when there is an increase in the average SNR.

When average SNR, $\bar{\gamma}=3$ the maximum capacity of the system is $16.6751 \mathrm{bits} / \mathrm{sec} / \mathrm{Hz}$, when average $\mathrm{SNR}, \bar{\gamma}=5$ the maximum capacity is $31.3864 \mathrm{bits} / \mathrm{sec} / \mathrm{Hz}$, at last, when $\bar{\gamma}=7$, maximum capacity is $47.2828 \mathrm{bits} / \mathrm{sec} / \mathrm{Hz}$.

It is clearly seen that the capacity of this SIMO channel is on the rise as average SNR of the system increases. But, the capacity of the system is the highest when the average SNR, $\bar{\gamma}=7$. So, the capacity can be improved when the average SNR of the system is considered higher.

\section{SIMO Channel Capacity with respect to the Instantaneous SNR}

In previous subsection it has been found that there is an improvement in the capacity of of the system when the average SNR is higher. So, in this subsection $\bar{\gamma}=7$ is considered for analysis the SIMO channel capacity behaviour with respect to the instantaneous SNR of the system. And the number of receiver antennas are considered, $n_{R}=2,4$ and 6 .

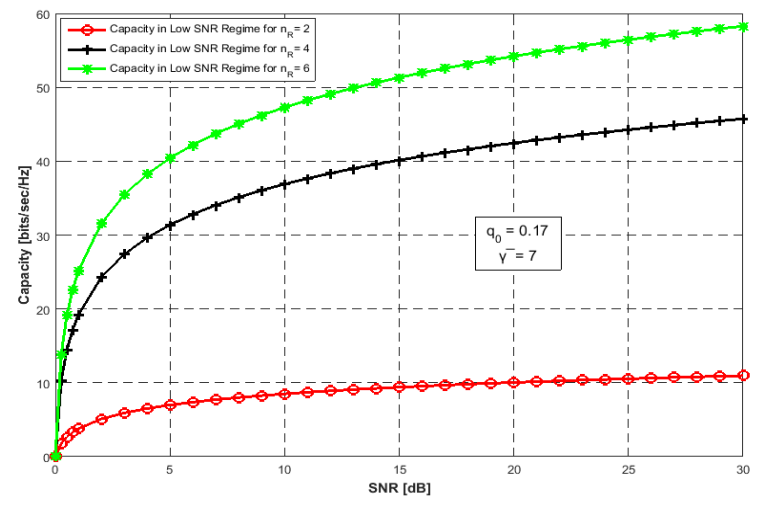

Fig. 4. SIMO Channel Capacity [bits/sec/Hz] with respect to the Instantaneous SNR [dB].

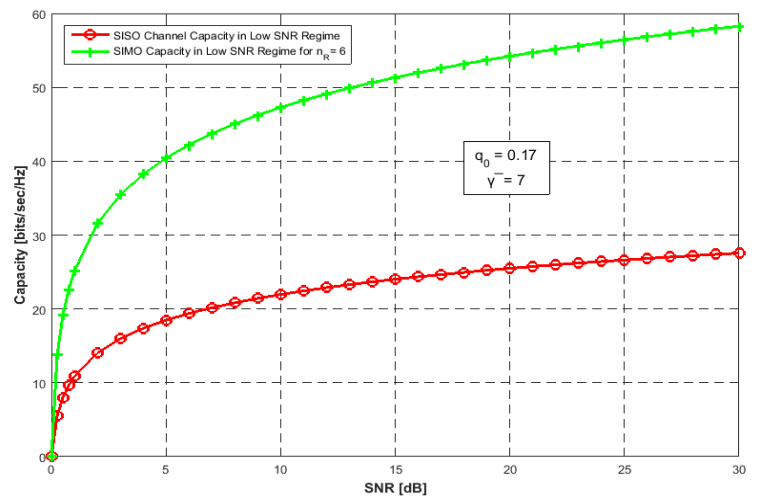

Fig. 5. Comparison of SISO Channel Capacity [14] and SIMO Channel Capacity

The increase of capacity with the increasing of instantaneous SNR for all the three numbers if receiver antennas can be observed from Fig. 4.

For receiver antenna, $n_{R}=2$, the maximum capacity when SNR is at $30 \mathrm{~dB}$ is $10.9601 \mathrm{bits} / \mathrm{sec} / \mathrm{Hz}$. When, the number receiver antennas, for $n_{R}=4$, the maximum capacity 
is $45.7309 \mathrm{bits} / \mathrm{sec} / \mathrm{Hz}$ and last, for $n_{R}=6$, the maximum capacity at $30 \mathrm{~dB}$ is $58.2786 \mathrm{bits} / \mathrm{sec} / \mathrm{Hz}$.

The capacity of this system is higher when there are more receiver antennas, in this case it highest when there are $n_{R}=6$ number of receiver antennas.

\section{Comparison of SIMO and SISO Channel Capacity}

In the previous subsection $\mathrm{C}$ it is found that when $n_{R}=6$ the capacity of this SIMO system is highest with respect to instantaneous SNR. So, in this subsection the SIMO channel capacity is considered for value of $n_{R}=6$.

The SISO channel capacity is taken with respect to instantaneous SNR from [14] for conducting the comparison with the SIMO channel capacity of this work.

In Fig. 5, the red line represents the maximum SISO channel capacity with respect to the instantaneous SNR [14] in low SNR regime. The green line in Fig. 5 represents the maximum SIMO channel capacity of this work also in the low SNR regime. Maximum channel capacity of the SISO channel is $27.5641 \mathrm{bits} / \mathrm{sec} / \mathrm{Hz}$ [14] and maximum capacity of SIMO channel observed in this work is $58.2786 \mathrm{bits} / \mathrm{sec} / \mathrm{Hz}$. There is about $111.43 \%$ increase in the capacity the SIMO system of this work.

So, it can be said that the capacity in SIMO system is much more higher that the capacity of SISO system.

\section{CONCLUSION AND FUTURE WORK}

In this work, the capacity of identically independently distributed Nakagami-q fading SIMO channel in low SNR regime is studied. For this study, the mathematical expression for the capacity in low SNR regime of this SIMO system is derived using small limit argument approximation. This paper presented in-depth analyses of the channel capacity of the SIMO wireless system. It has been found in this work that the capacity is increasing with the increasing of number of antennas at the receiver end and the capacity is higher when the average SNR of the system is higher. With respect to instantaneous SNR, it is seen that there is exponential growth in the capacity. The maximum capacity of this SIMO wireless channel is obtained when there are 6 number of receiver antennas at the receiver end in this case. From the comparison of the channel capacity between Nakagami-q fading SIMO and SISO wireless channels it has been found that the capacity of SIMO system outperforms the capacity of SISO system.

Though the derived equation can accurately measure the channel capacity, the measurement is only for low SNR regime. Moreover, as high SNR region is also an important part to consider when doing capacity performance analysis, the equations of this work can be re-used and modified to derive novel equation for high SNR region.

\section{REFERENCES}

[1] C. Shannon, "Communication theory of secrecy systems," Bell System Technical Journal, vol. 29, pp. 656-715, 1949.

[2] G. Foschini, "Layered space-time architecture for wireless communication in a fading environment when using multi-element antennas," Bell labs technical journal, vol. 1, no. 2, pp. 41-59, 1996.
[3] Arif Khan and Rein Vesilo, "A Tutorial on SISO and MIMO Channel Capacities," in Department of Electronics, Macquarie University NSW, Sydney Australia.

[4] T. Ratnarajah, "Secrecy capacity and secure outage performance for Rayleigh fading SIMO channel," in 2011 IEEE International Conference on Acoustics, Speech and Signal Processing (ICASSP), 2011.

[5] Shuai Ma, Ruixin Yang, Hang Li, Zhi-Long Dong, Huaxi Gu and Shiyin $\mathrm{Li}$, Achievable rate with closed-form for SISO channel and broadcast channel in visible light communication networks,"Journal of Lightwave Technology, vol. 35, no. 14, pp. 2778-2787, 2017.

[6] Nimay Ch Giri, Anwesha Sahoo, J. R. Swain, P. Kumar, A. Nayak and P. Debogoswami, "Capacity and performance comparison of SISO and MIMO system for next generation network (NGN)," International Journal of Advanced Research in Computer Engineering and Technology (IJARCET), vol. 3, no. 9, pp. 30131-3035, 2014.

[7] Wei Yang, Giuseppe Durisi, Tobias Koch and Yury Polyanskiy, "Quasistatic SIMO fading channels at finite blocklength," In 2013 IEEE International Symposium on Information Theory, pp. 1531-1535, 2013.

[8] Vignesh Sethuraman, Ligong Wang, Bruce Hajek and Amos Lapidoth, "Low-SNR capacity of noncoherent fading channels," IEEE Transactions on Information Theory, vol. 55, no. 4, pp. 1555-1574, 2009.

[9] B. Nkakanou., G. Y. Delisle, N. Hakem and Y. Coulibaly, "UWBSIMO channel capacity in an underground mine," In 2012 International Conference on Wireless Communications in Underground and Confined Areas, 2012.

[10] Ersen Ekrem and Sennur Ulukus, "The secrecy capacity region of the Gaussian MIMO multi-receiver wiretap channel," IEEE Transactions on Information Theory, vol. 57, no. 4, pp. 2083-2114, 2011.

[11] Sandro Adriano Fasolo and Renan Sthel Duque, "Fading channel simulator for Hoyt distribution,"in Instituto Nacional de Telecomunicacoes, Brasil.

[12] Romero-Jerez, Juan M. and F. Javier Lopez-Martinez, "A new framework for the performance analysis of wireless communications under hoyt (Nakagami-q) Fading," IEEE Transactions on Information Theory , vol. 63, no. 3, pp. 1693-1702, 2017.

[13] Romero-Jerez, Juan M. and F. Javier Lopez-Martinez, "Fundamental capacity limits of spectrum-sharing in Hoyt (Nakagami-q) fading channels," in In 2016 IEEE 84th Vehicular Technology Conference (VTCFall), 2016.

[14] Md. Mazid-Ul-Haque and Md. Sohidul Islam, "Data Rate Limit in Low and High SNR Regime for Nakagami-q Fading Wireless Channel," International Journal of Advanced Computer Science and Applications(IJACSA), 11(7), 2020.

[15] Nakagami and Minoru, "The m-distribution-A general formula of intensity distribution of rapid fading," in Statistical methods in radio wave propagation, Pergamon.

[16] Youssef, Neji, Cheng-Xiang Wang, and Matthias Patzold, "A study on the second order statistics of Nakagami-Hoyt mobile fading channels," IEEE Transactions on Vehicular Technology, 54(4), pp. 1259-65, 2005.

[17] K. S and Kölbig, "A definite integral with modified Bessel functions," Geneva.

[18] Simon, Marvin K., and Mohamed-Slim Alouini, "Digital communication over fading channels," New York.

[19] Amer M. Magableh and Mustafa M. Matalgah, "Capacity of SIMO systems over non-identically independent Nakagami-m channels," in 2007 IEEE Sarnoff Symposium, Princeton, NJ, 2007.

[20] J. G. Proakis, Digital Communication Systems, 4th edition, McGrawHill.

[21] Md Sohidul Islam and Mohammad Rakibul Islam, "Ergodic Capacity of a SIMO System Over Nakagami-q Fading Channel," DUET Journal, vol. 2, no. 1, 2014.

[22] Md Sohidul Islam and Mohammad Rakibul Islam, "Positive secrecy mutual information over non-identically independently distributed Nakagami-q fading wireless channel," in International Conference on Engineering, Research, Innovation and Education, 2013.

[23] Wolfram Research, Inc., Mathematica, Version 12.0, Champaign, IL (2019). 